\title{
Exploring the Use of Native Spider Silk as an Optical Fiber for Chemical Sensing
}

\author{
Kenny Hey Tow ${ }^{(0)}$, Member, OSA, Desmond M. Chow, Student Member, OSA, Fritz Vollrath, Isabelle Dicaire ${ }^{(1)}$, \\ Tom Gheysens ${ }^{(0)}$, and Luc Thévenaz ${ }^{(0)}$, Fellow, IEEE, Fellow, OSA
}

\begin{abstract}
A spider uses up to seven different types of silk, all having specific functions, as building material, weapon, and sensory organ to detect the presence of preys on its web. Recently, scientists have put under the limelight the extraordinary properties of this ancient material. Indeed, native silk, directly extracted from spiders, is a tough, biodegradable, and biocompatible thread used mainly for tissue engineering and textile applications. Blessed with outstanding optical properties, this protein strand can also be used as a bioresorbable optical fiber and is, moreover, intrinsically sensitive to chemical compounds. In this communication, the waveguiding properties of native dragline silk are assessed and a pioneering proof-of-concept experiment using pristine spider silk as an optical fiber to measure humidity content is demonstrated. The feasibility of using silk-based optical fiber chemical sensors is also discussed.
\end{abstract}

Index Terms-Chemical sensing, humidity sensor, optical fibre sensor, spider silk.

\section{INTRODUCTION}

$\mathbf{S}$ INCE fibre optic sensors (FOS) are becoming ubiquitous for monitoring purposes in natural and man-made infrastructures, there is a crying demand for researchers to develop innovative concepts that will allow FOS to go beyond their current state of the art as they are slowly but steadily reaching saturation in their performance [1]. FOS essentially exploit telecom silica optical fibres, for obvious practical and economic reasons, to benefit from their high quality mass production. In these systems, the fibres are used in a "passive" way and the sensing system exploit the effects natively present in the fibre, take advantage of their dependence on environmental quantities

Manuscript received July 31, 2017; revised September 8, 2017; accepted September 8, 2017. Date of publication September 25, 2017; date of current version February 24, 2018. (Corresponding author: Kenny Hey Tow.) This work was supported by the European Space Agency, under Ariadna study ${ }^{\circ} 14$ 6401. (Corresponding author: Kenny Hey Tow.)

K. Hey Tow, D. M. Chow, and L. Thévenaz are with the Group for Fibre Optics, SCI-STI-LT, Swiss Federal Institute of Technology of Lausanne, Lausanne CH-1015, Switzerland (e-mail: kenny.heytow@epfl.ch; desmond.chow@epfl.ch; luc.thevenaz@epfl.ch).

F. Vollrath is with the Department of Zoology, University of Oxford, Oxford OX1 3PS, U.K. (e-mail: fritz.vollrath@zoo.ox.ac.uk).

I. Dicaire was with the European Space Agency, Noordwijk 2201, The Netherlands., She is now with CCTT Optech, Montreal, QC H8N 2J4, Canada (e-mail: Isabelle.Dicaire@cctt-optech.ca).

T. Gheysens was with the European Space Agency, Noordwijk 2201, The Netherlands., He is now with Polymer Chemistry and Biomaterials Research Group, Ghent University, Ghent B-9000, Belgium (e-mail: tgheysens@ gmail.com).

Color versions of one or more of the figures in this paper are available online at http://ieeexplore.ieee.org.

Digital Object Identifier 10.1109/JLT.2017.2756095

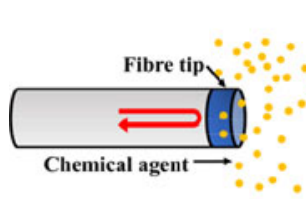

(a)

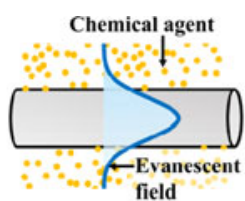

(b)

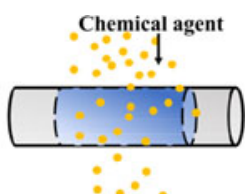

(c)
Fig. 1. Typical fibre-optic chemical sensors based on (a) fibre tip and (b) evanescent field sensing. (c) Simple transmission setup, based on a chemicallyreactive fibre, proposed in this communication.

and extract the information with a proper signal processing at the accuracy given by the natural response of the exploited effects. Therefore, silica fibres qualify as excellent candidates to monitor temperature change and axial strain but an improper material for detecting chemical species since silica is, by essence, chemically inert. Yet, an exhaustive list of fibre-optic chemical sensors (FOCS), which are silica optical fibre-based devices used to measure gases, vapours (hydrocarbons, organic solvents, etc.) and inorganic ions (e.g., to determine the $\mathrm{pH}$ level), is reported in the literature [2].

In fact, the optical fibre is reduced to, merely, the role of a convenient photon conveyor to bring light to and from a chemically-sensitive tip/coating and is not exploited as the sensing element in FOCS. For FOCS based on fibre-tip technology (see Fig. 1(a)), the optical fibre is used to convey light to indicators, doped in a thin film at the fibre end, which respond to the chemical species to be detected. These interactions may induce fluorescence, a quick and transient temperature or pressure change, etc. and can be monitored by a signature on the returned light. Since light interaction is confined to a restrictive sensing area, this class of FOCS do not take full advantage of the fibre sensing potentiality. Another conventional design for FOCS are systems based on evanescent field sensing as depicted in (see Fig. 1(b)). In such a configuration, the sensing mechanism relies on the interactions between the evanescent field of the guided light, the part of the light that is made to propagate out of the silica fibre, and the chemical species present in the vicinity of fibre's surface, usually coated with a chemically-sensitive material. The intensity of the evanescent field represents only a minute fraction of the total field, and although it can be enhanced by different methods, the gain in sensitivity is only incremental and the sensor is rendered much more complex if, for instance, localised surface plasmon resonance is employed [3]. Recently, an original indirect method based on optoacoustic probing in silica fibres was proposed to detect the presence of liquids in 
contact with the fibre [4]. The guided light optically activated transverse resonant acoustic waves, used as messengers to probe any change in the acoustic impedance mismatch between the fibre core and the cladding brought by the presence of a liquid on the fibre surface. However, this system is complex to set up and would probably still require a coating material for enhanced sensitivity or to detect gas.

One strategy to upscale the sensitivity of existing FOCS is to use other fibres or fibre devices, specially dedicated for this application. In this line of thought, the use of in-fibre gratings [5] and photonic crystal fibres [6] to measure chemical compounds have been mooted but these systems still require a coating (gold nanoparticles, polymer, etc.) for notable enhanced performance. To drastically improve the performance of FOCS, the optical fibre needs to play an active role as the sensing element. Recent improvements in the transparency and the fabrication process of Polymer Optical Fibres (POFs), have encouraged their use for optical fibre sensing [7]. Their intrinsic properties - high elastic strain limits, fracture toughness, flexibility, and potential negative thermo-optic coefficients-offer significant advantages for temperature and strain sensing-based applications. The hydrophilic properties of some POFs can also be exploited, for instance Poly (methyl methacrylate) (PMMA) based fibre Bragg gratings have been used for humidity sensing [8]. Natural protein fibres can prove to be another inspiring worth exploring since they are capable of interacting with the surrounding environment in their pristine condition. For instance, hydrophilic materials such as cellulose fibrils in wheat awns, horse hair, wool, etc. all swell during bulk water uptake [9]. These specific conformational changes lead to a signature change in material properties of the fibre bulk material, which can be measured by monitoring the parameters of light (intensity, phase, spectrum, polarisation, etc.) propagating along these natural threads. By collecting and analysing the transmitted light, the presence of the modifying agent can be detected using a very simple transmission setup. In this configuration, the optical fibre is both used as the light conveyor and the sensing element along its whole length. Therefore, the benefits of optical fibre sensing is entirely exploited since the guided light keeps fully confined in the fibre and the effect is accumulated along the fibre, thereby upscaling its sensitivity as shown in Fig. 1(c). Moreover, a transmission setup excludes the risk of any contamination by other unwanted agents or dust since light is strictly kept confined inside the fibre.

So far, the use of proteins in optical devices has been limited to thin film photosensitive deposits integrated in optical devices for optical logic gates [10], ultrafast photonic switching [11] and for bacteria analysis [12], but they have yet to be used as chemically-sensitive optical fibres. Silk is a very good material for our targeted application since the elemental building blocks of this material are exclusively proteins. Throughout history, silk fibres have mainly been used exclusively in the textile industry due to their natural sheen and distinctive smooth feel. Recent research in this field have shed light on the other extraordinary electrical [13], mechanical [14], [15] and biocompatible [16], [17] properties of this material, which can be naturally obtained from spiders or silkworms. Equally blessed with intrinsic optical properties, silk films made from fibres of Bombyx mori cocoons through a regeneration process, obtained by casting a silk fibron solution on an appropriate surface, have been used to make optical and photonic devices, for e.g., diffraction gratings [18], inverse opals [19], light-emitting transistors [20] and lasers [21], [22].

Silk threads can also be used as longitudinal waveguides, which could represent a new generation of mechanically strong, biocompatible and biodegradable optical fibres particularly suited for sensing applications. To obtain these light conveying fibres from cocoon silk, an additional processing step is required such as casting the silk fibroin solution into a stepindex optical waveguide [23] or a degumming process [24]. On the other hand, silk threads produced by spiders can guide light in their natural condition [25]. They are intrinsically sensitive to chemical species, and can be used, for instance, to measure ambient humidity level [26]. In this communication, the sensing mechanism involving conformational changes in silk protein structure to detect chemical species is briefly described. A detailed mechanical and optical characterisation of a pristine Nephilia edulis spider dragline silk is reported and an example of application of the silk fibre, as a breathalyser to monitor human expiration, is proposed.

\section{UNIQUE COMPOSITION OF SPIDER SILK}

The most attractive feature of spider silk lays in the composition of the protein fibres composing the silk. The amino acid composition of the dragline silk, secreted by the major ampullate glands of the spider and used by the latter as the main building material for its web, is composed mainly of the amino acids glycine $(30-40 \%)$ and alanine $(20-30 \%)$. The amount of glutamine (Glu) exceeds $10 \%$, while the amount of proline is about $5 \%$, indicating that the fibre is partly hydrophobic and partly hydrophilic [15]. A unique structural feature shared by silk protein sequences is their organisation into two types of alternating domains: i) a $\beta$-sheet block, composed of small repetitive units which tends to be hydrophobic and forms the crystalline part of the silk and ii) a non-repetitive hydrophilic part of the core sequence making up the amorphous regions mostly organized in $\alpha$-helices [27], [28]. A dragline silk can, therefore, be seen as a protein threads composed of repeating arrays of polypeptides containing both discrete crystalline and non-crystalline domains, oriented along a fibre axis. These two regions are held together into the fibre by reversible hydrogen bonds (see Fig. 2), which can be modified by different classes of molecules, making them ideal for detecting modifying agents such as water molecules, acids, bases, etc. Interacting molecules will either bind with the amino acids of the $\alpha$-helical portion; thereby affecting the elongation properties of the fibre or with amino acids of the $\beta$-sheet blocks, which will change the crystallinity of the fibre or the orientation of the crystalline blocks. These specific conformational reactions will all lead to changes in optical properties of the transmitted light along the silk fibre. Therefore, a multitude of compounds can be detected with only a single silk fibre as many different interaction modes are possible with the added benefit of potentially high sensitivity and quick response time since a $10-\mathrm{mm}$ sample of silk fibre is composed of several 


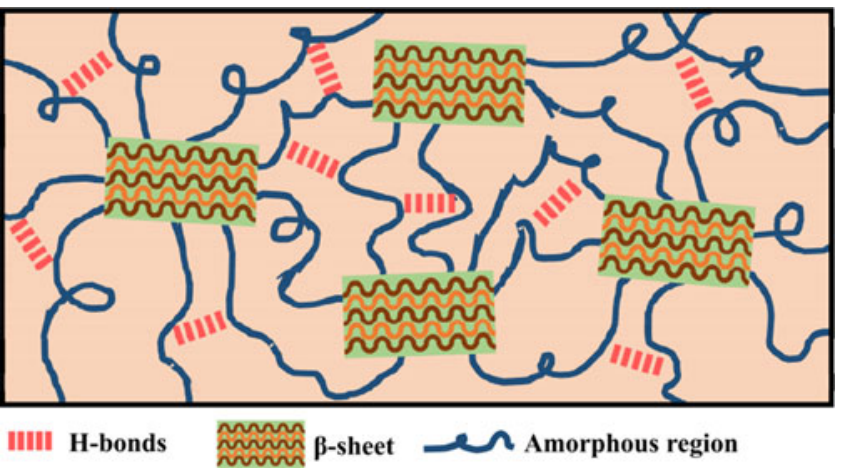

Fig. 2. Schematic representation of silk protein showing the $\beta$-sheets (crystalline region) embedded in the amorphous region. Reversible hydrogen bonds (red lines) maintain the orientation of the crystals along the fibre axis.

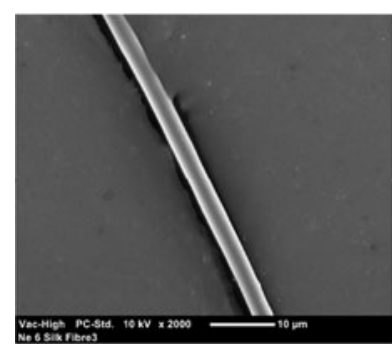

(a)

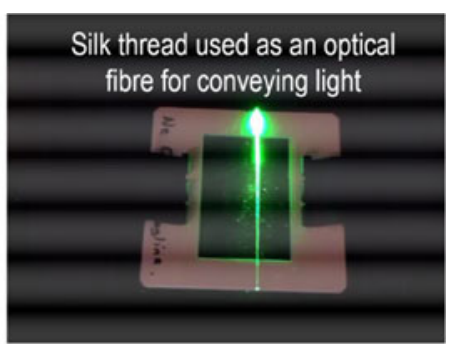

(b)
Fig. 3. (a) Scanning electron microscope (SEM) image of a dragline silk sample. (b) Light guiding achieved in a single strand of pristine dragline silk.

billions of these proteins, all capable of quickly binding to target molecules.

The amount of crystalline and amorphous regions in the fibre can be consistently tuned by changing the reeling speed and environmental conditions at which the silk it is spun. Reeling at low speed $(<1 \mathrm{~mm} / \mathrm{s})$ results in less crystalline fibres with thicker diameters and orientational order, i.e., polymer chain extension and longitudinal distribution of polarizability leading to higher values of birefringence. In contrast, enhanced chain orientation and lateral arrangement of polymer chains, resulting in a more crystalline fibre and increased transverse distribution of polarizability, can be achieved by increasing the reeling speed [29]-[31]. Thus, silk fibres can potentially be custom-made with a known amount of crystalline and amorphous regions to make it more/less sensitive to targeted chemical agents, such as humidity by tuning the percentage of the water-accessible $\beta$-sheets [32].

\section{SPIDER Silk ThreAdS AS ChEMICALly-SENSITIVE OPTICAL FIBRES}

Native dragline silk is obtained from the major ampullate glands of a female Nephila edulis, reeled from the same spider spigot onto a spool under controlled conditions at a constant speed of $5 \mathrm{~mm} / \mathrm{second}$. This results in a uniformly spun fibre with a smooth surface, circular cross-section and homogenous material properties, particularly adapted to be used as a waveguide (see Fig. 3(a)). The homogenous quality of the silk fibre sample has been confirmed by performing tensile tests and diameter measurements on three segments of the same fibre
TABLE I

Tensile Properties Indicating the Strain (\%), Initial Modulus $\left(\mathrm{E}_{\bmod }\right)$, BREAKING FORCE $\left(\mathrm{F}_{\max }\right)$ AND THE DIAMETER $(\mu \mathrm{M})$, MEASURED AT 3 Different Segments (SEg1, Seg2, SEg3) on the Same Fibre Sample

\begin{tabular}{lc|c|cc}
\hline \hline \multirow{2}{*}{ Parameter } & \multicolumn{3}{c}{ Measurement } & Average \\
\cline { 2 - 4 } & Seg 1 & Seg 2 & Seg 3 & \\
\hline Strain $(\%)$ & 33.81 & 34.22 & 34.59 & 34.2 \\
$\mathrm{E}_{\bmod }(\mathrm{MPa})$ & 9555.53 & 8355.22 & 8731.30 & 8880.7 \\
$\mathrm{~F}_{\max }(\mathrm{N})$ & 0.0232 & 0.0282 & 0.0271 & 0.026 \\
Diameter $(\mu \mathrm{m})$ & 5.30 & 5.63 & 5.78 & 5.6 \\
\hline \hline
\end{tabular}

TABLE II

Measured Material and Optical Properties of the Dragline Silk SAMPLE USED AS OPTICAL FIBRE

\begin{tabular}{lc}
\hline \hline Parameter & Value \\
\hline Fibre diameter & $5.6 \mu \mathrm{m}$ \\
Refractive index & $1.54(635 \mathrm{~nm})$ \\
Reeling speed & $5 \mathrm{~mm} / \mathrm{s}$ \\
Strain & $34.2 \%$ \\
Initial modulus & $8880.7 \mathrm{MPa}$ \\
Transmission window & Visible $-1360 \mathrm{~nm}$ \\
Propagation losses & \\
$\quad$ Visible & $0.4 \mathrm{~dB} / \mathrm{mm}$ \\
$\quad$-band & $\sim 0.9 \mathrm{~dB} / \mathrm{mm}$ \\
Birefringence & $10^{-3}-10^{-2}$ \\
\hline \hline
\end{tabular}

sample at the following positions on the reel; one at the beginning (Seg1), one in the middle (Seg2) and one at the end of the reel (Seg3). The data for our silk sample is tabulated in Table I.

The refractive index of silk being around 1.55 [33], light guiding can be achieved by total internal reflection. Fig. 3(b) shows light propagation along a $25 \mathrm{~mm}$ pristine dragline silk sample, placed on a holder, with a standard end-fire injection with 3-axes translational stages. When stored in dry, dust-free conditions at room temperature, no apparent degradation was noticed on the sample and light guiding could still be achieved one year later with the same losses. The measured optical properties of our silk sample is tabulated in Table II. Fig. 4(a) represents the measured transparency window of our silk sample, obtained by launching supercontinuum light and comparing the input-output transmission spectrum into the same sample. The transparency window of our silk thread is limited to around $1360 \mathrm{~nm}$, restricting the use of this fibre in the telecommunications wavelength $(1500 \mathrm{~nm})$. The silk fibre's propagation loss was measured on several silk samples using an image-based distributed scattering loss technique [34]. Fig. 4(b) and (c) are examples of measured relative intensity of scattered light as a function of position along the silk strand at two different wavelengths, in which the propagation losses are estimated from the gradients of fitted linear curve. Diffusion spots, which leads to excessive light scattering, are clearly spotted and their contribution neglected in the determination of propagation losses. Measurements have been performed on several samples and, on average, a propagation losses of $0.4 \pm 0.2 \mathrm{~dB} / \mathrm{mm}$ and $0.9 \pm 0.2 \mathrm{~dB} / \mathrm{mm}$ were obtained respectively in the visible and the O-band wavelength regions. These values coincide with the $1.05 \mathrm{~dB} / \mathrm{mm}$ at 900 


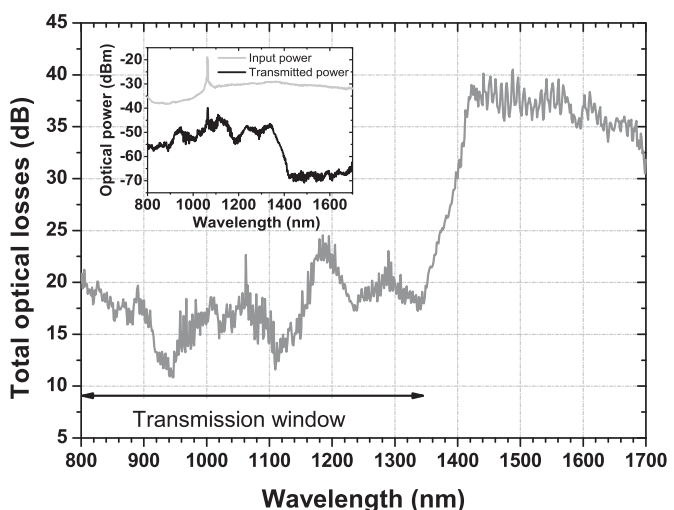

(a)

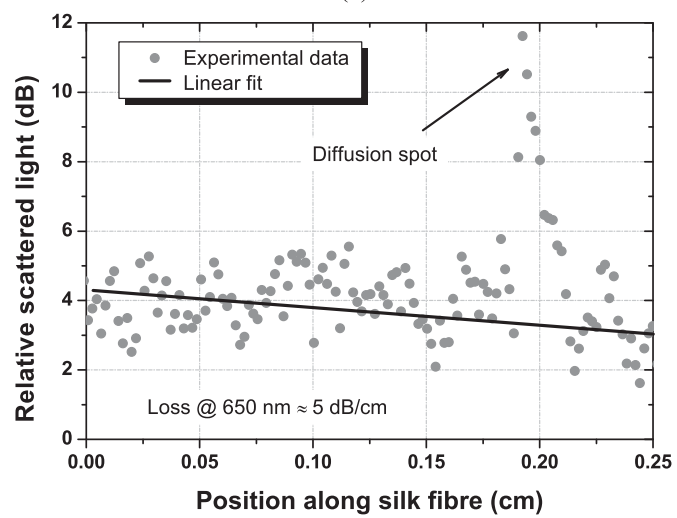

(b)

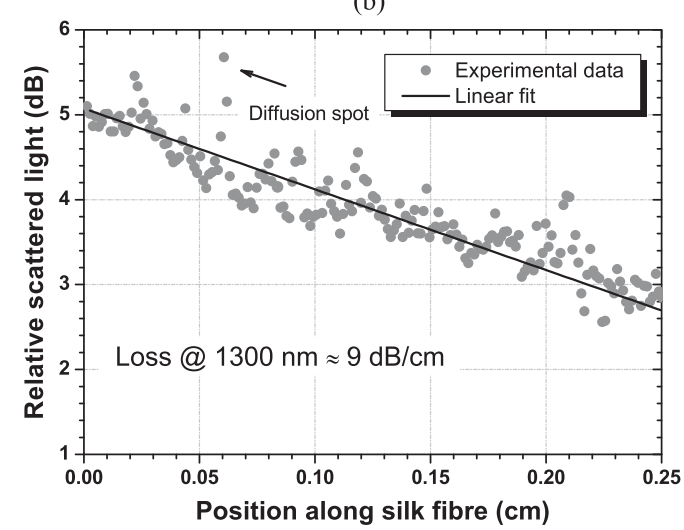

(c)

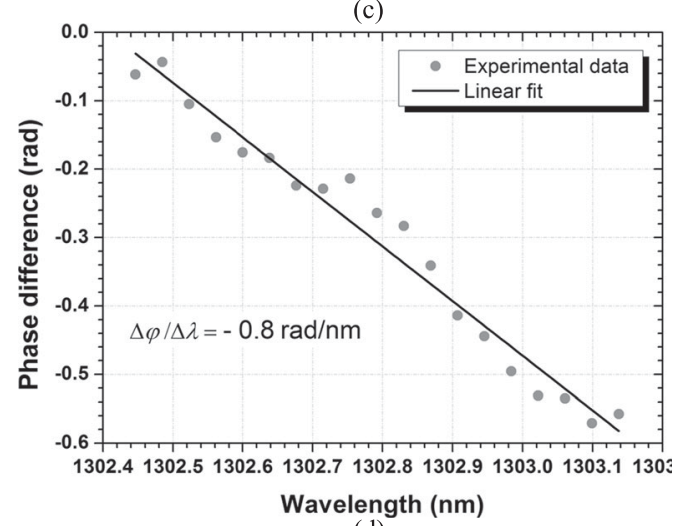

(d)

Fig. 4. Optical characterisation of a dragline silk sample, used as an optical fibre, in terms of: (a) transparency window; propagation losses (b) in the visible spectrum and (c) in the $1300 \mathrm{~nm}$ wavelength region using image-based analysis; and (d) birefringence. nm reported in native Nephila clavipes spider silk [25] and is one order of magnitude lower than in degummed silkworm silk fibres [24]. Finally, the birefringence of our silk thread was investigated using a wavelength scanning method [35]. Laser light in the O-band was launched inside the silk fibre and the state of polarization (SOP) of the transmitted light measured using a polarization analyser. Detuning the wavelength of the input light induces a change in the phase difference $\varphi$ between the two orthogonally polarized modes of the transmitted light, as shown in Fig. 4(d). The measurement was repeated with several samples and the retrieved birefringence values are in the order of $10^{-3}-10^{-2}$ at $1302 \mathrm{~nm}$, assuming a negligible spectral dependence of the birefringence and, thus, considering phase and group birefringence as identical. This value is three to four orders of magnitude higher than in silica fibres and is due to the ordered array of protein (high density crystalline and amorphous sections) along the silk fibre [28].

\section{Relative Humidity Fibre-Optic Sensor Based on SPIDER SILK}

Modifying agents with different chemical properties break hydrogen bonds in the silk material [36], which can affect the birefringence of the silk thread [37]. For instance, exposure to humidity results in infiltration of water molecules into the silk material. They interact with the hydrophilic amino acids in the amorphous region, bind themselves to the random coils and break the relatively weak hydrogen bonding holding together the protein threads. This relaxes the silk [38] without changing the overall molecular orientation. This process is highly reversible and upon drying, water molecules are lost only from the random coil region until the humidity exceeds a critical threshold of $\sim 70 \%$, beyond which the silk supercontracts [39]. At this point, the water molecules start reacting with the molecular bonding between the protein molecules, which permanently change the material properties of silk.

Fig. 5(a) schematically represents the polarimetric setup, used to validate our proposed concept of using silk fibre as a humidity sensor. It consists in launching linearly polarised light at $1300 \mathrm{~nm}$ into a $25 \mathrm{~mm}$-long dragline silk sample via end-fire injection and monitoring the SOP of the collected light under the influence of varying humidity conditions. To exclude the influence of varying external ambient conditions (such as temperature, pressure variations) on our results, the silk strand is placed in a gas chamber, maintained under controlled pressure (MKS Baratron pressure sensor) and temperature (PT1000 temperature sensor) during the whole experiment. The silk fibre being kept at the same reeling tension and held at the two ends, it swells when exposed to humidity since it is refrained from contracting along its length. This results in a change in the silk fibre's geometry in its whole volume, which brings a substantial change in the SOP of the output signal as shown in Fig. 5(b): a rotation with a phase of $\Delta \varphi$ on the Poincaré sphere could be detected as soon as a small amount of water vapour is introduced inside the chamber. Interestingly enough, the presence of a non-polar gas did not seem to affect the silk fibre's birefringence. Fig. 5(c) shows the change in the SOP of the output 


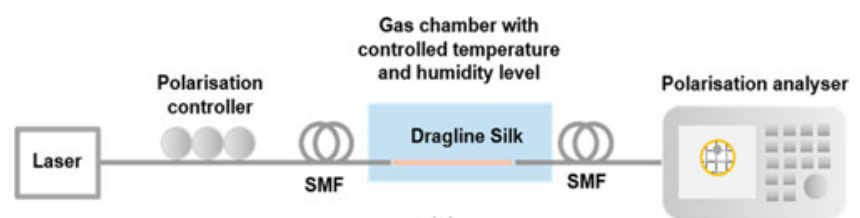

(a)

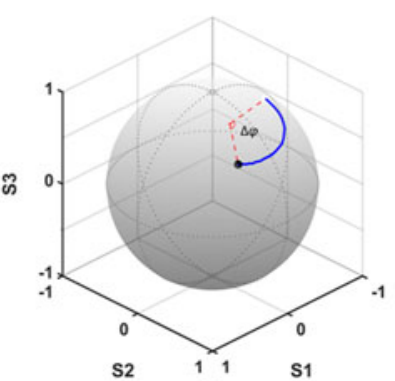

(b)

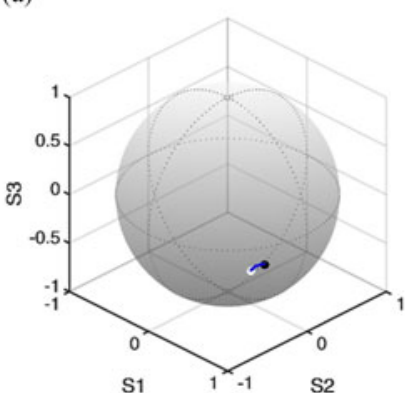

(c)
Fig. 5. (a) Polarimetric setup used in our experiment. Polarisation transformations on a Poincaré sphere of the output light when the silk thread is exposed to (b) a small amount of water vapour and (c) a non-polar gas $\left(\mathrm{CO}_{2}\right) . S M F$; Single mode fibre; White dot: initial SOP; Black dot: final SOP; blue line: SOP transformation path.

light recorded when pure $\mathrm{CO}_{2}$ gas was flushed in the gas chamber. This suggests that native silk could be used to distinguish between chemical species having different polarities.

To calibrate the system, the gas chamber was hermetically sealed and the relative humidity (RH) level inside was measured with a $\mathrm{HC} 1000$ humidity sensor (E+E Electronik). When the environment reaches equilibrium condition, i.e., a constant RH level of around $40 \%$ measured inside the gas chamber, the evolution of the SOP of the output light was monitored on the polarization analyser for 500 seconds. Only a negligible drift of $0.02 \pi \mathrm{rad}$ was recorded for $\Delta \varphi$ during this measurement time.

The sensor was evaluated over a relative humidity level range from 40 to $65 \%$ to avoid working in the supercontraction regime. First, water vapor was gradually introduced into the gas chamber to gradually increase the RH level in the chamber. The corresponding change in $\Delta \varphi$ was measured and plotted in Fig. 6(a). A linear response with a sharp slope of $\sim 0.7 \pi \mathrm{rad} / \% \mathrm{RH}$, which reflects the high sensitivity over this range of the sensor was observed. To check the reversibility of the process in this range, the gas chamber was opened and desiccant was added to reduce the humidity level back to $40 \%$. A similar sensitivity of $\sim-0.7 \pi \mathrm{rad} / \% \mathrm{RH}$ was obtained (see Fig. 6(b)).

One appealing application for our proposed silk-based fibre optical sensor is for air and breath analysis. A proof-of-concept experiment, whereby the silk fibre is used to monitor human exhaled air, has been performed. The peaks on Fig. 7. represent detected SOP phase change caused by exhaled air detected from a subject's nose $20 \mathrm{~cm}$ away from the sensor; no air is expelled in the first 7 seconds, followed by two long exhalations and, finally, short and periodically nose-expelled air. Since human expiration is mainly composed of water vapour, we can assume that the silk sensor is essentially detecting a change in relative humidity with a very good response time. It is worth noting that desorption rate is lower than absorption rate in our silk sensor since the latter takes slightly more time to get back to equilibrium

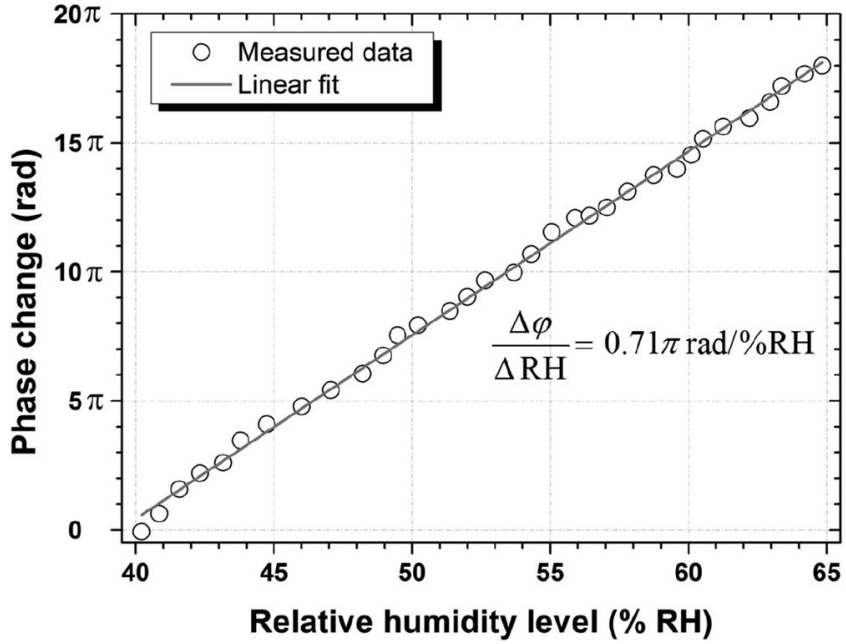

(a)

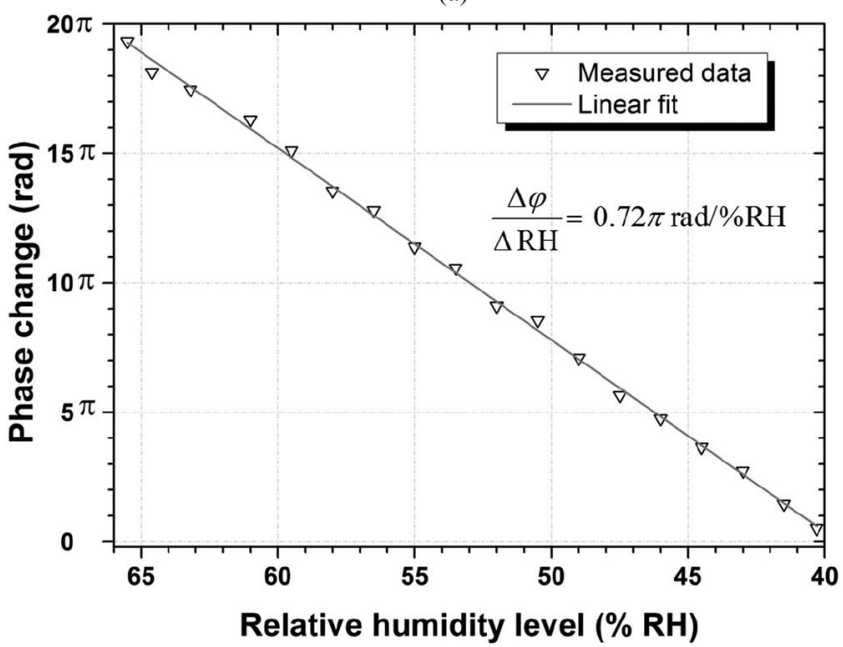

(b)

Fig. 6. Measured phase change when the relative humidity level is (a) increased from 40 to $65 \%$ and (b) reduced from 65 to $40 \%$ at a temperature of 24 ${ }^{\circ} \mathrm{C}$.

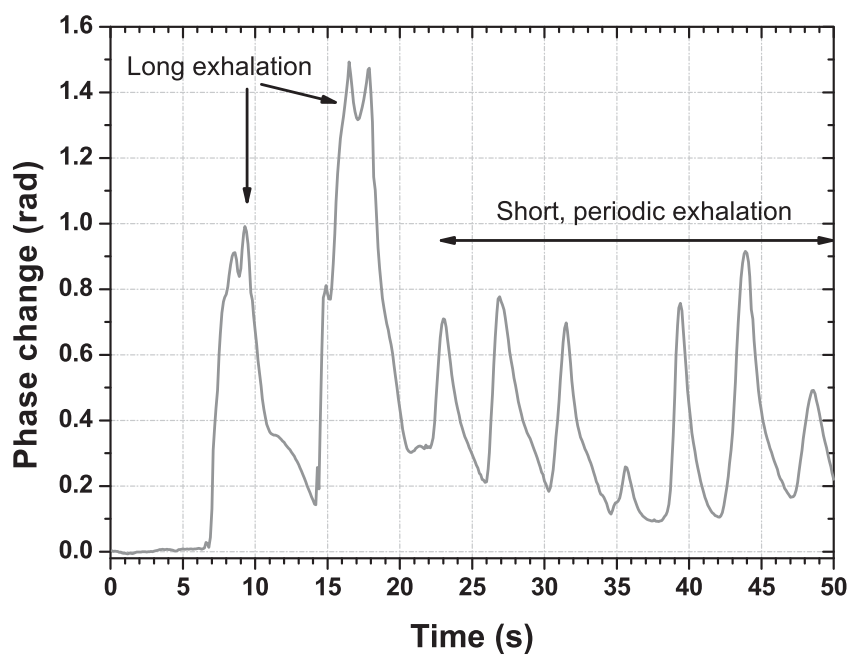

Fig. 7. Phase change of light through silk fibre due to nose-exhaled breaths (humidity level change). 
humidity condition. This result paves the way towards the use of silk-inspired threads for breath analysis. If the silk strands can be made exclusively selective to individual breath biomarkers, they can represent a faster, more compact, and economic alternative to the widely used laser spectroscopic detection techniques for breath analysis [40] or to detect volatile organic compounds (VOCs) level for air quality monitoring.

\section{DISCUSSION}

Native silk, directly woven by spiders, offers remarkable optical properties and can intrinsically guide light within a transparency window extending from the visible wavelength to around $1400 \mathrm{~nm}$ with propagation losses below $1 \mathrm{~dB} / \mathrm{mm}$ and under relatively high birefringence. These properties allowed us using silk as a light conveying, chemically-sensitive protein thread as a fibre-optic sensor to measure ambient relative humidity content.

Advantages of silk-based fibre optic sensing are numerous: high sensitivity, fast response time, sensitive to polar chemical compounds. Having a complex molecular structure, the presence of other classes of chemical compounds in the vicinity of silk brings selective conformational reactions leading to different changes in optical properties of light guided along the silk fibre. These protein threads could potentially be used in a similar way as described in Section IV for sensing other polar target agents in trace amounts such as ammonia, ethanol, VOCs, etc. [37] and probably represent a much better alternative for fibre-optic chemical sensing than conventional glass fibres since the latter usually require coatings, even to be used as relative humidity sensors [41]. Another route worth exploring is to associate the waveguiding properties of spider silk fibres with the Raman silk spectrum [42] to detect conformational changes in the molecular structure due to modifying agents. Raman shifts are of the same order of magnitude as that observed in silica fibres, corresponding to $\sim 100 \mathrm{~nm}$ at near IR wavelengths [36], thus measurable using a typical high-resolution optical spectrum analyser.

Silk threads also offer a crucial degree of freedom. First of all, their material properties (degree of crystallinity, sensitivity to humidity, etc.) can be intrinsically modified during the spinning process performed at ambient conditions. Moreover, silk has the unique property to be drawn under ambient conditions (temperature, pressure), so that dyes and specific colorimetric reagents into the bulk material can potentially be incorporated in the silk bulk material, which would otherwise be denatured at the very high drawing temperatures of $\sim 2000{ }^{\circ} \mathrm{C}$ for silica fibres, giving potential access to functionalised fibre sensors, exploiting the countless possibilities offered by chemistry for selective sensing.

All these qualities make these protein threads very promising for future applications. However, along this "silk road" there are still many barriers to overcome for us to fully exploit this highly promising material for optical fibre sensing. While native silk is suitable for proof-of-concept, artificially fabricated silk will be needed for upscaling to industry level. Hopefully, the recent progress achieved in the understanding of the silk proteins and the drawing of artificial silk [43] will allow the production of tailor-made low-loss synthetic optical threads. Ideally, this would be combined with an ability to fabricate functionalised fibres for selective sensing of specific chemical compounds. This would represent the first building blocks towards the conception of a new generation of less energy-consuming, biocompatible, selective sensitive devices through a novel bulk protein sensing approach.

\section{REFERENCES}

[1] L. Thévenaz, "Next generation of optical fibre sensors: New concepts and perspectives," Proc. SPIE, vol. 9157, pp. 9157AN-1-9157AN-4, 2014.

[2] X.-D. Wang and O. S. Wolfbeis, "Fiber-optic chemical sensors and biosensors (2013-2015)," Analytical Chem., vol. 88, no. 1, pp. 203-227, 2015.

[3] B. Kuswandi, R. Andres, and R. Narayanaswamy, "Optical fibre biosensors based on immobilised enzymes," Analyst, vol. 126, no. 8, pp. 1469-1491, 2001.

[4] Y. Antman, A. Clain, Y. London, and A. Zadok, "Optomechanical sensing of liquids outside standard fibers using forward stimulated brillouin scattering," Optica, vol. 3, no. 5, pp. 510-516, 2016.

[5] T. Yeo, T. Sun, K. Grattan, D. Parry, R. Lade, and B. Powell, "Characterisation of a polymer-coated fibre Bragg grating sensor for relative humidity sensing," Sens. Actuators B, Chem., vol. 110, no. 1, pp. 148-156, 2005.

[6] A. Hassani and M. Skorobogatiy, "Design of the microstructured optical fiber-based surface plasmon resonance sensors with enhanced microfluidics," Opt. Exp., vol. 14, no. 24, pp. 11616-11621, 2006.

[7] Y. Koike and K. Koike, "Progress in low-loss and high-bandwidth plastic optical fibers," J. Polymer Sci. Part B, Polymer Phys., vol. 49, no. 1, pp. 2-17, 2011 .

[8] W. Zhang and D. J. Webb, "Humidity responsivity of poly (methyl methacrylate)-based optical fiber Bragg grating sensors," Opt. Lett., vol. 39, no. 10, pp. 3026-3029, 2014.

[9] D. E. Meyer, "Miniature moisture sensors for in-package use by the microelectronics industry," in Proc. 1975 13th Annu. Rel. Phys. Symp., 1975 pp. 48-52.

[10] A. Dér, S. Valkai, A. Mathesz, I. Andó, E. K. Wolff, and P. Ormos, "Protein-based all-optical sensor device," Sens. Actuators B, Chem., vol. 151, no. 1, pp. 26-29, 2010.

[11] L. Fábián et al., "Protein-based ultrafast photonic switching," Opt. Exp., vol. 19, no. 20, pp. 18861-18870, 2011.

[12] A. Mathesz et al., "Integrated optical biosensor for rapid detection of bacteria," Optofluidics, Microfluidics Nanofluidics, vol. 2, no. 1, pp. 1521, 2015.

[13] C. Müller, M. Hamedi, R. Karlsson, R. Jansson, R. Marcilla, and O. Inganäs, "Woven electrochemical transistors on silk fibers," Adv. Mater. vol. 23, no. 7, pp. 898-901, 2011.

[14] J. Gosline, P. Guerette, C. Ortlepp, and K. Savage, "The mechanical design of spider silks: From fibroin sequence to mechanical function," J. Exp. Biol., vol. 202, no. 23, pp. 3295-3303, 1999.

[15] F. Vollrath and D. P. Knight, "Liquid crystalline spinning of spider silk," Nature, vol. 410, no. 6828, pp. 541-548, 2001.

[16] H. Wendt et al., "Artificial skin-culturing of different skin cell lines for generating an artificial skin substitute on cross-weaved spider silk fibres," PloS One, vol. 6, no. 7, p. e21833, 2011.

[17] P. Domachuk, H. Perry, J. J. Amsden, D. L. Kaplan, and F. G. Omenetto, "Bioactive self-sensing optical systems," Appl. Phys. Lett., vol. 95, no. 25, p. 253702, 2009.

[18] F. G. Omenetto and D. L. Kaplan, "A new route for silk," Nature Photon., vol. 2, no. 11, pp. 641-643, 2008.

[19] V. M. Swinerd, A. M. Collins, N. J. Skaer, T. Gheysens, and S. Mann "Silk inverse opals from template-directed $\beta$-sheet transformation of regenerated silk fibroin," Soft Matter, vol. 3, no. 11, pp. 1377-1380, 2007.

[20] R. Capelli et al., "Integration of silk protein in organic and light-emitting transistors," Organic Electron., vol. 12, no. 7, pp. 1146-1151, 2011.

[21] S. Toffanin et al., "Low-threshold blue lasing from silk fibroin thin films," Appl. Phys. Lett., vol. 101, no. 9, p. 091110, 2012.

[22] R. R. da Silva et al., "Silk fibroin biopolymer films as efficient hosts for DFB laser operation,” J. Mater. Chem. C, vol. 1, no. 43, pp. 7181-7190, 2013.

[23] M. B. Applegate, G. Perotto, D. L. Kaplan, and F. G. Omenetto, "Biocompatible silk step-index optical waveguides," Biomed. Opt. Exp., vol. 6 , no. 11, pp. 4221-4227, 2015. 
[24] S. Kujala, A. Mannila, L. Karvonen, K. Kieu, and Z. Sun, "Natural silk as a photonics component: A study on its light guiding and nonlinear optical properties," Sci. Rep., vol. 6, 2016, Art. no. 22358.

[25] N. Huby et al., "Native spider silk as a biological optical fiber," Appl. Phys. Lett., vol. 102, no. 12, p. 123702, 2013.

[26] K. H. Tow, D. M. Chow, F. Vollrath, I. Dicaire, T. Gheysens, and L. Thévenaz, "Towards a new generation of fibre-optic chemical sensors based on spider silk threads," in Proc. 2017 25th Opt. Fiber Sens. Conf., 2017, pp. 1-4.

[27] C. Y. Hayashi and R. V. Lewis, "Molecular architecture and evolution of a modular spider silk protein gene," Science, vol. 287, no. 5457, pp. 1477-1479, 2000 .

[28] K. Mita, S. Ichimura, and T. C. James, "Highly repetitive structure and its organization of the silk fibroin gene," J. Mol. Evol., vol. 38, no. 6, pp. 583-592, 1994.

[29] Z. Shao and F. Vollrath, "Materials: Surprising strength of silkworm silk," Nature, vol. 418, no. 6899, pp. 741-741, 2002.

[30] B. Madsen, Z. Z. Shao, and F. Vollrath, "Variability in the mechanical properties of spider silks on three levels: Interspecific, intraspecific and intraindividual," Int. J. Biolog. Macromol., vol. 24, no. 2, pp. 301-306, 1999.

[31] C. Holland, K. O'Neil, F. Vollrath, and C. Dicko, "Distinct structural and optical regimes in natural silk spinning," Biopolymers, vol. 97, no. 6, pp. 368-373, 2012.

[32] F. Paquet-Mercier, T. Lefèvre, M. Auger, and M. Pézolet, "Evidence by infrared spectroscopy of the presence of two types of $\beta$-sheets in major ampullate spider silk and silkworm silk," Soft Matter, vol. 9, no. 1, pp. 208-215, 2013.

[33] D. J. Little and D. M. Kane, "Image contrast immersion method for measuring refractive index applied to spider silks," Opt. Exp., vol. 19, no. 20, pp. 19182-19189, 2011.

[34] Y. Okamura, S. Yoshinaka, and S. Yamamoto, "Measuring mode propagation losses of integrated optical waveguides: A simple method," Appl. Opt., vol. 22, no. 23, pp. 3892-3894, 1983.

[35] K. Kikuchi and T. Okoshi, "Wavelength-sweeping technique for measuring the beat length of linearly birefringent optical fibers," Opt. Lett., vol. 8, no. 2, pp. 122-123, 1983.

[36] Z. Shao and F. Vollrath, "The effect of solvents on the contraction and mechanical properties of spider silk," Polymer, vol. 40, no. 7 , pp. 1799-1806, 1999.

[37] K. H. Tow, D. M. Chow, F. Vollrath, I. Dicaire, T. Gheysens, and L. Thévenaz, "Spider silk: A novel optical fibre for biochemical sensing," in Proc. Int. Conf. Opt. Fibre Sens., 2015, pp. 96347-D1-96347-D4.

[38] J. Guan, F. Vollrath, and D. Porter, "Two mechanisms for supercontraction in nephila spider dragline silk," Biomacromolecules, vol. 12, no. 11, pp. 4030-4035, 2011.

[39] T. A. Blackledge et al., "How super is supercontraction? Persistent versus cyclic responses to humidity in spider dragline silk," J. Exp. Biol., vol. 212, no. 13, pp. 1981-1989, 2009.

[40] C. Wang and P. Sahay, "Breath analysis using laser spectroscopic techniques: Breath biomarkers, spectral fingerprints, and detection limits," Sensors, vol. 9, no. 10, pp. 8230-8262, 2009.

[41] T. Yeo, T. Sun, and K. Grattan, "Fibre-optic sensor technologies for humidity and moisture measurement," Sens. Actuators A, Phys., vol. 144, no. 2, pp. 280-295, 2008.

[42] T. Lefèvre, F. Paquet-Mercier, J.-F. Rioux-Dubé, and M. Pézolet, "Structure of silk by Raman spectromicroscopy: From the spinning glands to the fibers," Biopolymers, vol. 97, no. 6, pp. 322-336, 2012.

[43] A. Rising and J. Johansson, "Toward spinning artificial spider silk," Nature Chem. Biol., vol. 11, no. 5, pp. 309-315, 2015.

Kenny Hey Tow received the M.Sc. degree from the University of Montpellier II, Montpellier, France, in 2009, and the Ph.D. degree in physics from the University of Rennes 1, Rennes, France, in February 2013. His Ph.D. research work, performed at FOTON Laboratory, Lannion, France, was focused on developing Brillouin lasers, made from microstructured chalcogenide fibere, and the study of their noise properties. In 2013, he joined the Group for Fibre Optics, Swiss Federal Institute of Technology of Lausanne, Lausanne, Switzerland. His current research focuses on specialty fibers (photonic crystal fibere, chalcogenide, and polymer fibers, etc.) for the development of new optical fiber sensors and laser sources. He is a Member of the Optical Society of America.
Desmond M. Chow received the B.Eng. degree in electrical engineering and the M.Sc. Eng. degree in photonics from the University of Malaya, Kuala Lumpur, Malaysia, in 2011 and 2014, respectively. He is currently working toward the $\mathrm{Ph} . \mathrm{D}$. degree in photonics at Swiss Federal Institute of Technology of Lausanne, Lausanne, Switzerland. His research interests include acoustooptic effect, distributed optical fiber sensors, nonlinear fiber optics, plasmonic biosensors, and bandgap fibers.

Fritz Vollrath, biography not available at the time of publication

Isabelle Dicaire received the Ph.D. degree from Swiss Federal Institute of Technology in Lausanne, Lausanne, Switzerland, where she developed an expertize in fiber optics sensors for the aerospace industry.

In 2012, she joined the Advanced Concepts Team, European Space Agency, as a Research Fellow. She is currently an Innovation and Operations Manager with CCTT Optech, Montreal, QC, Canada. Her current responsibilities include project management tasks for several $R \& D$ projects as well as resource management and oversight for all Optech projects. Her professional activities included suggesting and performing several interdisciplinary research projects, session chairing at several international conferences in the field, as well as recruitment of personnel.

Tom Gheysens, biography not available at the time of publication.

Luc Thévenaz (M'01-SM'12-F'17) received the M.Sc. and Ph.D. degrees in physics from the University of Geneva, Geneva, Switzerland.

In 1988, he joined Swiss Federal Institute of Technology of Lausanne, Lausanne, Switzerland, where he currently leads a research group involved in photonics, namely fiber optics and optical sensing. He achieved with his collaborators the first experimental demonstration of optically controlled slow and fast light in optical fibers, realized at ambient temperature and operating at any wavelength since based on stimulated Brillouin scattering. He also contributed to the development of Brillouin distributed fiber sensing by proposing innovative concepts pushing beyond barriers. During his career, he was with Stanford University, with Korea Advanced Institute of Science and Technology, with Tel Aviv University, with the University of Sydney, and with the Polytechnic University of Valencia. In 2000, he cofounded the company Omnisens that is developing and commercializing advanced photonic instrumentation based on distributed fiber sensing. He is an Associate Editor in three major scientific journals. His research interests include Brillouin-scattering fiber sensors, slow and fast light, nonlinear fiber optics, and laser spectroscopy in gases.

Dr. Thévenaz is a Fellow of the Optical Society of America. 